\title{
CONTRIBUCIÓN AL DESARROLLO TERRITORIAL DE LOS GRUPOS DE PRODUCTORES RURALES PERTENECIENTES AL PROGRAMA CAMBIO RURAL DEL INTA COLONIA BENITEZ
}

\section{Contribution to rural producers' territorial development from "Cambio Rural" program by INTA Colonia Benitez}

\author{
Rusas, Víctor A.; Vagabculov, Javier R.; Rosello, José E.; Sáez, Roberto A.; Gómez, \\ Viviana D.; Geijo, Rubén A.; Ondo Misi, Sergio G. \\ Instituto Nacional de Tecnología Agropecuaria. Estación Experimental Agropecuaria Colonia Benítez. \\ Dirección postal: Marcos Briolini sin N. CP: 3505. E-mail: rusas.victor@inta.gob.ar
}

\section{RESUMEN}

El programa Cambio Rural del Ministerio de Agricultura Ganadería y Pesca de la Nación Argentina es una herramienta de extensión rural e intervención territorial que promueve la mejora productiva y socioeconómica de los pequeños y medianos productores rurales, siendo el Instituto Nacional de Tecnología Agropecuaria (INTA) el responsable de su coordinación operativa y ejecución presupuestaria. El objetivo de este trabajo es analizar los resultados obtenidos por los grupos pertenecientes a la Estación Experimental Agropecuaria (EEA) Colonia Benítez del INTA, durante el periodo febrero 2018 a marzo 2020. La estrategia utilizada en los grupos es el asociativismo, con la metodología de trabajo grupal y la asistencia técnica profesional. En este sentido, el método grupal se sustenta en los siguientes pilares: La reunión mensual, el plan de trabajo grupal, el plan de mejoras individuales y la visita individual predial. La mayoría de los productores integrantes de los grupos afianzaron sus fortalezas, accedieron a detectar los puntos débiles de sus actividades, abordaron problemas y oportunidades, promovieron el trabajo asociado y aprovecharon la asistencia del Promotor Asesor, el Agente de Proyecto y del Equipo Técnico de extensión e investigación del INTA Colonia Benítez. En los grupos quedaron instalados los logros comunes, a través del asociativismo, mediante el cual se incorporaron tecnologías adaptadas a cada actividad, se comercializaron productos y servicios, se disminuyeron costos y se agregó valor obteniendo nuevos productos, servicios y procesos. Otro punto logrado, es la vinculación institucional e interinstitucional que permitió articular acciones a nivel municipal, provincial y nacional; tanto con privados y organismos gubernamentales.

Palabras clave: Asociativismo; Intervención; Desarrollo Territorial; Metodología Grupal.

\begin{abstract}
The "Cambio Rural" Program from the Argentine Ministry of Livestock, Agriculture and Fisheries is a tool for rural extension and territorial intervention that promotes productive and socioeconomic improvement of small and medium-sized rural producers, being Nacional Institute of Agricultural Technology (INTA) in charge of coordination and budget execution. The aim of this work is to analyze the results obtained by groups of producers working with Colonia Benítez Agricultural Experiment Station (EEA) INTA, from February 2018 to March 2020 . The implemented strategy among the groups is associativism along with workgroup methodology and professional technical assistance. In this sense, the group method is based on: monthly meetings, the group workplans, individual development plans, individual visits to the lots. Most of the producers from the groups reinforced their strengths, agreed to detect the weaknesses in their activities, introduced their problems and opportunities, fostered associated work and took advantage from the assistance provided by the Counselor, the Project Officer and Colonia Benítez INTA technical extension and investigation team. Common achievements were installed through associativism through which technologies adapted to each activity were incorporated, products and services were commercialized, costs were reduced, and value was added to new products, services and processes. Another point achieved is the institutional and inter-institutional connection that allowed articulating actions at local, provincial and national levels; both with private and governmental organizations.
\end{abstract}

Key words: Associativism; Intervention; Territorial development; Workgroup Methodology.

Recibido: 19/jun/2020. Aceptado: 21/jul/2020 


\section{INTRODUCCIÓN}

El Programa Cambio Rural (CR) se creó en el año 1993 desde la Secretaría de Agricultura, Ganadería y Pesca de la Nación. Actualmente, lo gestiona el Ministerio de Agricultura, Ganadería y Pesca de la Nación; siendo el Instituto Nacional de Tecnología Agropecuaria (INTA) el responsable de su coordinación operativa y ejecución presupuestaria a nivel territorial por medio de sus 56 Estaciones Experimentales Agropecuarias (EEA) distribuidas en todo el territorio argentino. La finalidad del mismo es, por medio de la asistencia técnica y el método de trabajo grupal promover y facilitar la intensificación y/o reconversión productiva, como un medio para mejorar la situación productiva y socioeconómica de los pequeños y medianos productores rurales. En este trabajo, pondremos especial énfasis en la gestión del Programa Cambio Rural como estrategia de intervención territorial, con el objetivo de analizar los resultados obtenidos, desde febrero del 2018 hasta marzo del 2020, por los grupos de productores pertenecientes a la Estación Experimental Agropecuaria (EEA) Colonia Benítez del INTA.

La EEA Colonia Benítez, se ubica en la zona sureste de la Provincia del Chaco, en la localidad de Colonia Benítez, situada a unos $28 \mathrm{~km}$ de la capital de la provincia (Resistencia), núcleo poblacional de más de 450.000 habitantes, lo que la convierte en un gran centro de consumo de productos. El área geográfica de cobertura incluye los departamentos San Fernando, Primero de Mayo, Bermejo, Libertad, Tapenagá y Gral. Dónovan. En estos, se distribuyen las Agencias de Extensión Rural (AERs) de Basail, Makalle y Las Palmas, y las Oficinas de Desarrollo Rural (ODRs) de Colonia Benítez y de Gestión Metropolitana en el gran Resistencia; todas de gestión INTA.

La zona, se caracteriza por contar con bajos, esteros, cañadas, monte y selva de ribera (Boletín RIAN Chaco -Formosa, 2014). A su vez, estas se clasifican según su cobertura vegetal en monte, selva de ribera, pastizales, esteros y cañadas (Morello y Adámoli, 1974). Las principales actividades agropecuarias y de servicios desarrolladas son: la ganadería (bovina, porcina, aviar y piscícola), la fruti-horticultura (hortalizas de hoja y pesadas), la apicultura (producción de miel) y el turismo rural. Los productores, son del estrato de pequeña a mediana escala, los cuales tienen tradición productiva y presentan en general ciertos problemas, pudiéndose mencionar a los relacionados con la eficiencia productiva y la comercialización. Respecto a la gestión del programa, éste mantiene una estructura funcional conformada por un Coordinador Nacional, un Coordinador Territorial y Agentes de Proyecto (AP) los que interactúan con INTA y su estructura programática a través de las Plataformas de Innovación Territorial (PIT), y con la estructura funcional de la institución, como las Direcciones, las Coordinaciones de Extensión e Investigación, los Jefes de Agencias de Extensión Rural (AERs) y Oficinas de Desarrollo Territorial (ODRs). Los AP, interactúan con los técnicos Promotores Asesores de grupos (PA) del sector privado y con los referentes de los grupos de productores.

Desde la EEA Colonia Benítez, se gestionan a través de sus AERs y ODRs 16 (dieciséis) grupos de productores: 7 (siete) ganaderos, 1 (uno) porcino, 1(uno) piscícola, 2 (dos) hortícolas, 1(uno) apícola y 4 (cuatro) de turismo rural. Estos, están integrados entre 8 a 12 productores con actividades, escala y problemáticas similares, y son asistidos por un técnico Promotor Asesor privado. La metodología de trabajo grupal, plantea la realización de diagnósticos, la planificación de actividades, el seguimiento, monitoreo y la evaluación. Las herramientas utilizadas para el desarrollo grupal son: la capacitación, el asesoramiento técnico especializado, el intercambio de experiencias, el acceso al financiamiento y la organización de actividades de vinculación con actores territoriales como municipios, universidades, empresas, cooperativas, etc.

En general, los problemas a nivel grupal se relacionan con la organización predial, la utilización de herramientas de gestión empresarial, el uso de registros, la incorporación de tecnología productiva, la imposibilidad para cubrir económicamente la asistencia técnica de un profesional calificado, el acceso a fuentes financieras, la competitividad en la comercialización y el desarrollo de procesos de agregado de valor en origen. Por último, y puntualizando en las actividades de la zona, mencionamos los problemas más recurrentes a los siguientes: AEn la ganadería: inconvenientes para la incorporación de mejoras al manejo del rodeo dado por la infraestructura deficitaria; los bajos índices reproductivos e índices de producción de carne por hectárea dados por la alta carga animal y falta de planificación; inconvenientes en la gestión económica, entre otros.; B- En la frutihorticultura: se dan tanto problemas de organización (escaso volumen y continuidad en la oferta, acceso al crédito, gestión económica, planificación) como estructurales: bajo nivel de implementación de tecnología de riego, 
manejo de suelos, cobertura de cultivos, implementación de buenas prácticas, etc. Esto, impacta fuertemente y tiene efecto en la competitividad (baja calidad lograda) y en las acciones comerciales asociativas para abastecer mercados de cercanía; C- En la apicultura: en la zona hay aproximadamente unos 80 apicultores que realizan su actividad dependiendo netamente de la oferta de néctar y polen en un ambiente nativo, siendo los problemas más comunes la incorporación de tecnologías en sanidad, nutrición, genética y gestión de la empresa; D- En el turismo rural: la actividad plantea problemas en función a cuestiones como: conectividad, señalización, infraestructura operativa, acceso al crédito, estrategias de marketing, y el bajo número de visitantes a los establecimientos.

\section{METODOLOGÍA}

El trabajo junto al Programa Cambio Rural se realiza implementando una metodología de trabajo en grupos de productores.

\section{Que se considera un grupo:}

Se sabe que existen diversas definiciones de grupo, por lo cual en este trabajo se adapta la enunciada por (Rivière 1985): "Un grupo es un conjunto restringido de personas, que interactúa mediante diferentes roles para lograr un objetivo común, mediante una tarea pre-establecida en el Plan de Trabajo Grupal”. En el Programa Cambio Rural, cada grupo está conformado por al menos ocho integrantes, para asegurar una cierta heterogeneidad de visiones y experiencias; pero no más de doce, a fin de facilitar la participación de todos sus miembros y posibilitar de esta manera, que el promotor asesor cumpla con las visitas a los establecimientos.

\section{Estrategia implementada}

La estrategia implementada en el Programa Cambio Rural para promover el desarrollo es el "asociativismo", mediante una metodología de trabajo grupal con asistencia técnica profesional. De esta manera, el método de trabajo consistente en el trabajo grupal se sustenta en los siguientes pilares (Manual Operativo Cambio Rural Ministerio de Agroindustria de la Nación Secretaría de Agricultura):

\section{La reunión}

Es el momento en el cual el grupo manifiesta todo su potencial de creatividad y trabajo, donde se intercambian ideas y experiencias. Esta instancia es enriquecedora y abre un abanico de alternativas a los miembros, manteniendo la libertad de decisión individual y grupal. La reunión se debe realizar sistemáticamente una vez por mes.

\section{El Plan de Trabajo Grupal (PTG)}

El plan es la expresión del proyecto grupal, describiendo los problemas/oportunidades comunes de los miembros del grupo y definiéndose los objetivos que el mismo se proponga, los resultados esperados, la estrategia para alcanzarlos, las actividades a realizar y los indicadores para medir y evaluar su cumplimiento. El PTG, constituye una herramienta fundamental para guiar la tarea del Promotor Asesor de grupo, analizado por el Agente de Proyecto y la estructura funcional de la EEA quiénes trasmitirán preguntas y sugerencias de mejora. El proceso de formulación es de seis meses con un horizonte de planeamiento de tres años.

\section{El Plan de Mejoras Individual (PMI)}

Cada productor debe confeccionar con su Promotor Asesor (PA) el PMI, que es la herramienta de planificación individual que permite al PA conocer la situación inicial del productor, y consensuar con él la forma de alcanzar la situación deseada. Debe realizarse en no más de tres meses desde el alta del grupo. A su vez, cada establecimiento cuenta con un "Cuaderno de campo" dónde, además de los datos del productor, grupo, promotor y Agente de proyecto se coordinan cada una de las visitas mensuales al establecimiento y detalles de cada reunión, como así también el seguimiento de la actividad.

\section{Metodología Enfoque de Marco Lógico (EML)}

Utilizado para elaborar los PTG y PMI, el EML facilitó el proceso de conceptualización, diseño, ejecución y evaluación de planes de trabajo. El mismo, está centrado en la orientación por objetivos, la orientación hacia grupos beneficiarios y en el facilitar la participación y la comunicación entre las partes interesadas. 
El EML, se utilizó en todas las etapas de formación de los planes grupales: en la identificación y valoración de actividades que encajen en el marco de los planes, en la preparación del diseño de los mismos de manera sistemática y lógica, en la implementación de proyectos y para el seguimiento, monitoreo y evaluación. (Ortegón et al., 2015).

De modo general, se la propuesta consiste en realizar un resumen del proceso de desarrollo en una matriz, que integra los elementos antes mencionados. Dicha matriz es conocida como la Matriz del Proyecto (MP).

En este sentido, junto a los Promotores Asesores, se trabajó en el seguimiento de actividades y en instancias de capacitación y talleres. Así mismo, se realizó una primera instancia de monitoreo, de cual se obtuvo el estado de situación de las actividades con una descripción de las fortalezas y debilidades para el primer año de funcionamiento. Esta información, nos muestra el grado de avance hacia los objetivos y los resultados esperados en el medio término. El proceso completo de seguimiento, monitoreo y evaluación busca fortalecer a los grupos a través de una reflexión compartida, que posibilite el aprendizaje y la mejora continua.

\section{Herramientas de soporte a la metodología de trabajo}

La principal herramienta de soporte es la asistencia técnica grupal e individual y la formación continua de sus actores (Figura 1). Ésta, es una actividad continua del programa y está destinada tanto a los productores como a los Agentes de proyecto y Promotores asesores. Los programas de capacitación, se incluyen en un Plan Operativo Anual de Actividades (POA). Durante estos últimos dos años de gestión del programa, se organizaron 20 espacios de capacitación, en su mayoría del tipo técnico productivos, dos dedicados a la gestión de empresas y tres de metodología. De éstos, siete fueron en temática ganadera, cinco en turismo rural, tres en producción fruti-hortícola, dos en apicultura, dos de fortalecimiento a los promotores y una dirigida a peones rurales. En este sentido, participaron 1012 personas entre productores, técnicos y estudiantes. El monto económico invertido en las capacitaciones ascendió a un total de $\$ 1.982 .500$ y lo aportado por el programa CR fue de $\$$ $951.200,00$ (más de $\$ 1.000 .000$ de pesos aportaron otras entidades del medio).

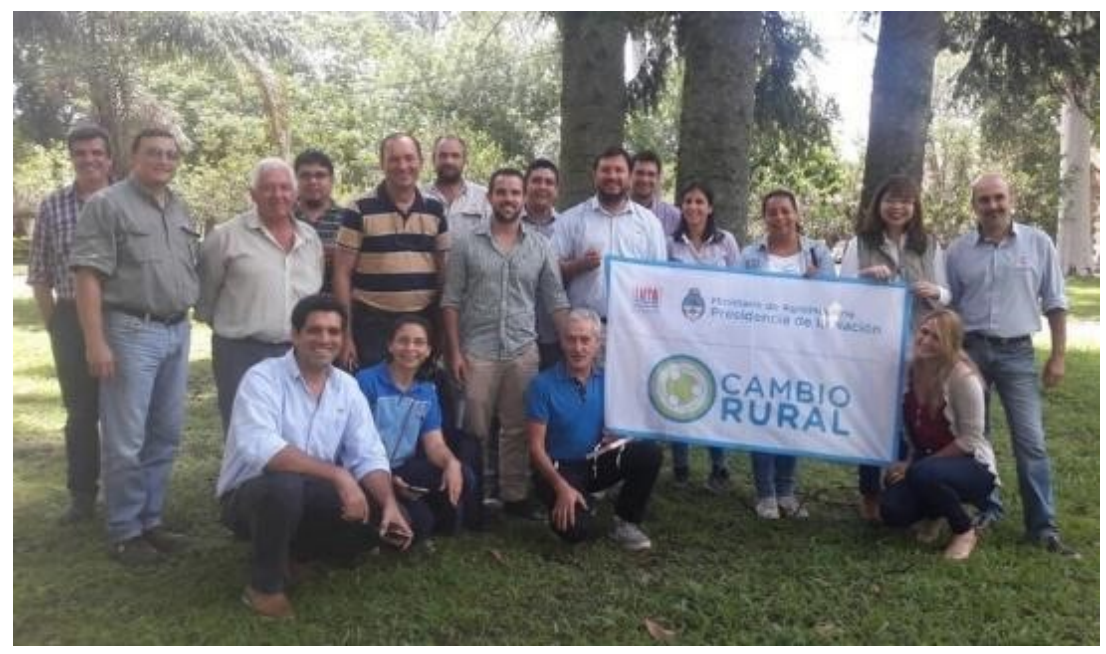

Figura 1. Taller de capacitación para promotores asesores.

\section{RESULTADOS}

\section{Estrategia asociativa del programa}

En el año 2017, se presentó la propuesta de conformación de unos 25 grupos para su aprobación en la coordinación Nacional del programa Cambio Rural. Luego del proceso de evaluación y de cumplimento administrativo, se logra la aprobación de 16 grupos en diversas temáticas productivas, de los cuales y al momento del trabajo, 13 grupos cumplieron con dos años de ejecución de PTG (empezando en febrero de 2018 el primer año y culminando el segundo año en marzo del 2020).

Estos grupos, nuclean un total de 128 productores y un equipo de 12 Promotores Asesores. Los mismos, se distribuyen geográficamente en las AERs y ODRs de la EEA Colonia Benítez del INTA, de la siguiente manera (Tabla 1): 
Rusas, V.A. et al.: Desarrollo territorial de grupos de productores rurales.

Tabla 1. Grupos conformados en las distintas actividades productivas por localidad.

\begin{tabular}{cclll}
\hline Actividad & AER Basail & AER Makalle & AER Las Palmas & \multicolumn{1}{c}{$\begin{array}{c}\text { ODR Colonia } \\
\text { Benítez }\end{array}$} \\
\hline Ganaderos & $\begin{array}{l}\text { 1.Colonia Pastoril } \\
\text { 2. Dos Provincias } \\
\text { 3.Charadai }\end{array}$ & 4.Vaca Porá & 6. Bermejo & 10. $1^{\circ}$ de Mayo \\
\hline Hortícolas & 5.Integración & & 11.Viento Norte \\
\hline Turismo Rural & & $\begin{array}{l}\text { 7.Rincones de un } \\
\text { Humedal } \\
\text { 8.Turismo a Cam- } \\
\text { po y Rio }\end{array}$ & $\begin{array}{l}\text { 12.Ecoturismo Chaco } \\
\text { Experiencia Natural } \\
\text { 13. Confluencia Isla } \\
\text { del Cerrito. } \\
\text { 14. Picadas del Tacú }\end{array}$ \\
\hline Apícola & & & $\begin{array}{l}\text { 15.Humedales } \\
\text { Saludables }\end{array}$ \\
\hline Piscícola & & 9.Peces del Chaco & \\
\hline Porcinos & & & 16.Porcinos del Este \\
\hline
\end{tabular}

A continuación, se observa la distribución de productores por actividad en los 16 grupos aprobados (Figura 2).

\section{Distribución de Productores por actividad}

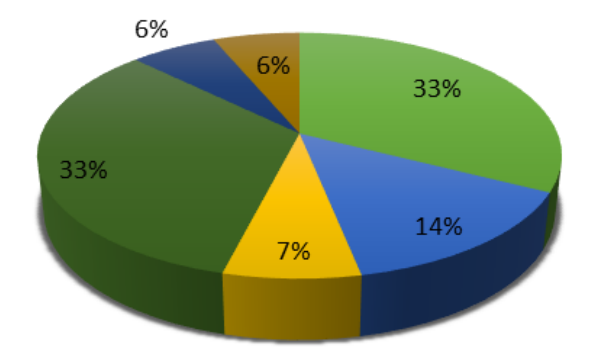

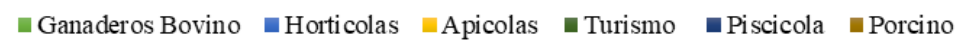

Figura 2. Distribución de productores agrupados por actividad (\%).

\section{Metodología de trabajo y planificación grupal}

La Coordinación de Cambio Rural Nacional a través de su comité evaluador aprueba en el año 2018 el alta definitiva de 16 grupos de Cambio Rural lo que permite disponer de un presupuesto para cubrir aportes económicos de los PA y los gastos operativos del grupo, para tres años de trabajo.

La etapa siguiente a esto, fue la elaboración de 16 Planes de Trabajo Grupales (PTG) y 128 Planes de Mejora Individuales (PMI). Esta etapa, fue crucial y demandó un proceso de trabajo conjunto, donde cada plan fue revisado y aprobado por estructura funcional del programa, interviniendo el agente de proyecto, los jefes de AERs y ODRs, la dirección de EEA, la dirección regional de INTA y las coordinaciones del Programa Cambio Rural tanto regional como nacional.

En la Figura 3, se presenta el avance en la ejecución de los PTG en función a los indicadores socio productivos pre establecidos en las planillas de actividades y cronograma de trabajo a corto, mediano y largo plazo realizadas durante los años 2018 al 2020. 


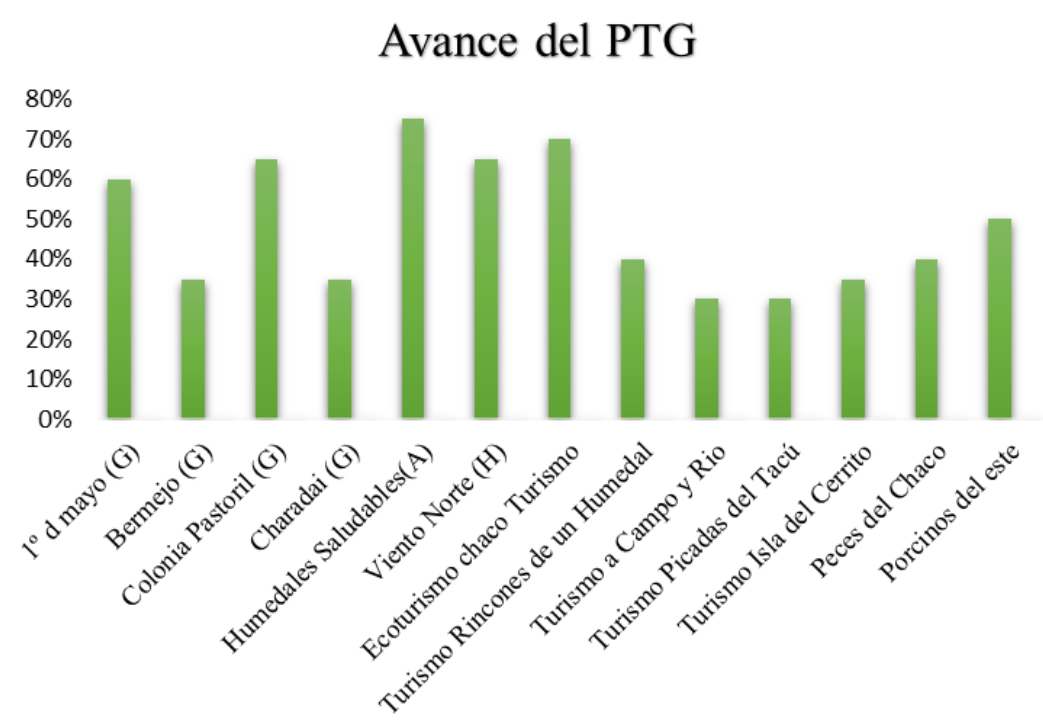

Figura 3. Situación de avance (\%) de cada Plan de Trabajo Grupal (PTG) para los dos primeros años de ejecución desde su aprobación formal (2018-2020).

En el análisis de los 13 grupos que culminaron el segundo año de avance de PTG, se observa que cinco grupos $\left(1^{\circ}\right.$ de Mayo, Colonia Pastoril, Humedales saludables, Viento Norte y Eco Turismo Chaco) superan el $60 \%$ de avance de ejecución, destacándose el grupo apícola Humedales Saludables, el cual supera el $70 \%$ de avance en su PTG. Esta situación, nos demuestra que los mismos lograron pasar la primera etapa de grupo denominada "conocimiento y confianza", pasando luego a la etapa natural de "consolidación grupal"; donde pudieron capitalizar la asistencia técnica brindada por los Promotores asesores, permitiendo esto, un avance superior en cuanto a gestión y ejecución de actividades. Respecto al resto, en estos 8 (ocho) grupos, la ejecución del PTG en promedio es de un $40 \%$ debido a distintas circunstancias que retrasaron la realización de las actividades planificadas, tales como la renuncia de promotores asesores, los periodos de inundación acaecidos, la falta de profesionales formados en actividades no tradicionales (como el turismo rural y la piscicultura), la distancia y ubicación de los emprendimientos, entre otras.

Aquí, se observa que el grupo Porcinos del Este se ubica en un $50 \%$ de avance de su PTG, logrado por la autogestión del mismo, aun ante la renuncia del técnico promotor asesor. Es preciso mencionar, que este grupo, se apoyó fuertemente en técnicos de la ODR Colonia Benítez, lo que le permitió fortalecer la interacción grupal y dar un gran paso en su organización y consolidación asociativa mediante la conformación de una cooperativa.

En cuanto al grupo Peces del Chaco, solo alcanza un 40\% de ejecución de su PTG debido principalmente a la desvinculación de su promotor asesor, lo cual produjo un aletargamiento del grupo. Recién cuando se pudo incorporar un nuevo profesional con la formación requerida, se lograron grandes avances como compras en conjunto que abarataron costos, mejoras en la nutrición y alimentación de los peces, implementación de nuevas técnicas de cosecha y de comercialización.

En cuanto a la actividad de los grupos de turismo rural, cuatro logran un avance de PTG de entre un 35 al 40 $\%$. Entre estos grupos, encontramos a Rincones de un humedal, Turismo Isla del Cerrito, Picadas del Tacú, Turismo a campo y Rio. Este bajo nivel de ejecución del plan, se debió principalmente al periodo de inundación transitado en la zona y a la desvinculación del promotor asesor. Aquí, el problema fue encontrar técnicos locales formados en la temática, principalmente por la ubicación de los grupos en zona rural del departamento Bermejo.

Por último, el grupo ganadero Bermejo alcanza el 35\% de ejecución de su PTG, debido al periodo de inundación prolongado dado en la zona, lo que imposibilito la normal ejecución de las actividades planificadas.

\section{Análisis por actividad productiva grupal}

Los resultados obtenidos en los grupos de productores ganaderos (Figura 4) fueron los siguientes: 
El 85\% mejoró su comercialización, realizando ventas en conjunto (Figura 5).

El 80\% tuvo acceso a alguna línea crediticia en ese periodo. Esto favoreció la inversión en infraestructura (alambrados, aguadas, boyeros eléctricos) en el $78 \%$ de los establecimientos vinculados al programa.

El 73\% implantó pasturas, mejorando la disponibilidad de alimento para el ganado.

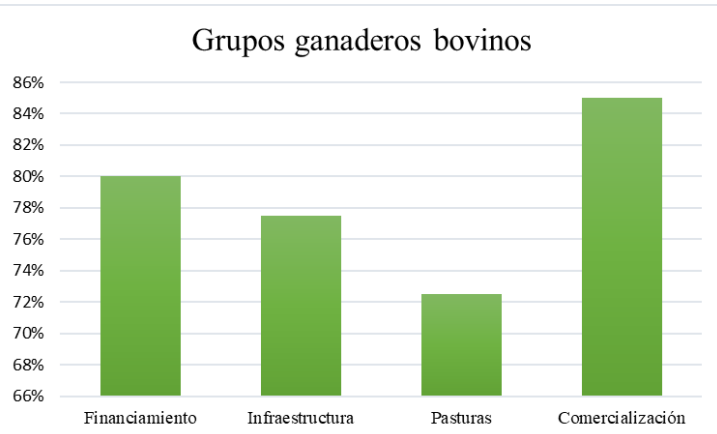

Fuente: Elaboración propia, a partir de datos PA Mata, N; Zapata, G; Mosci, D

Figura 4. Productores que mejoraron la comercialización, implantación de pasturas, infraestructura y accedieron a financiamiento (sobre 60 productores).

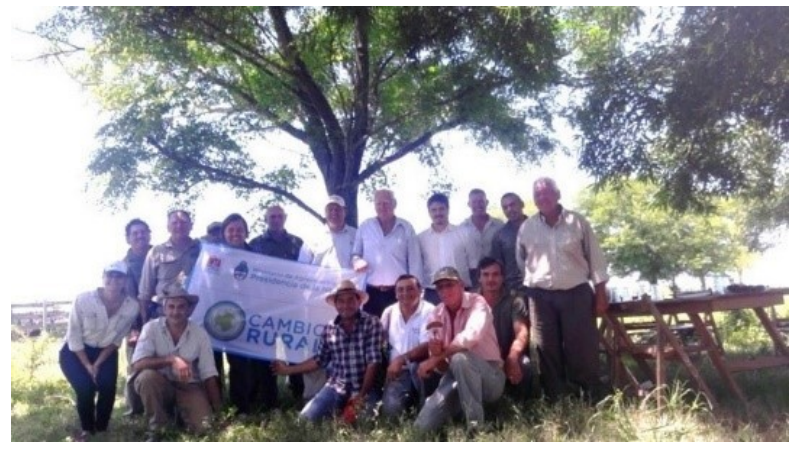

Figura 5. Reunión mensual de organización comercial Grupo ganadero Primero de Mayo.

Los resultados obtenidos en los grupos de productores fruti-hortícolas (Figura 6) fueron los siguientes:

El 50\% accedieron a nuevos canales de comercialización.

El 25\% incursionaron en procesos de agregado de valor.

El 88\% incorporaron las Buenas Prácticas Agrícolas en sus establecimientos.

El 38\% tuvo acceso a las líneas de financiamiento.

El 100\% incorporó el cultivo de frutilla, diversificando su producción, y se integraron al sistema cooperativo (Figura 7).

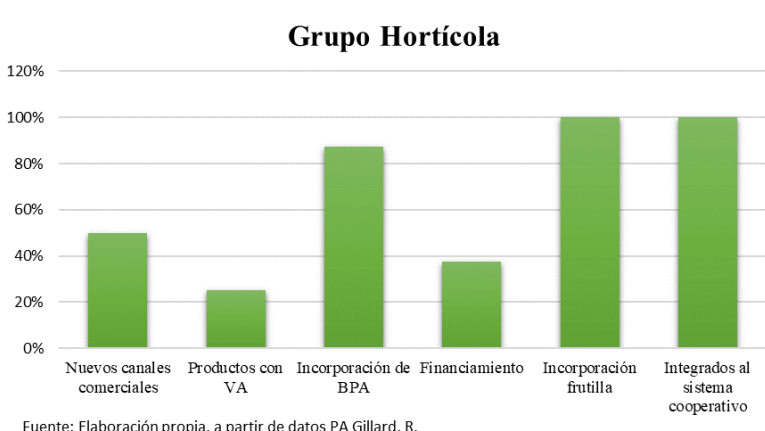

Figura 6. Productores que mejoraron la comercialización, valor agregado, BPA, accedieron a financiamiento, incorporaron frutilla y se integraron al sistema cooperativo ( $\%$ de un total de 8 productores).

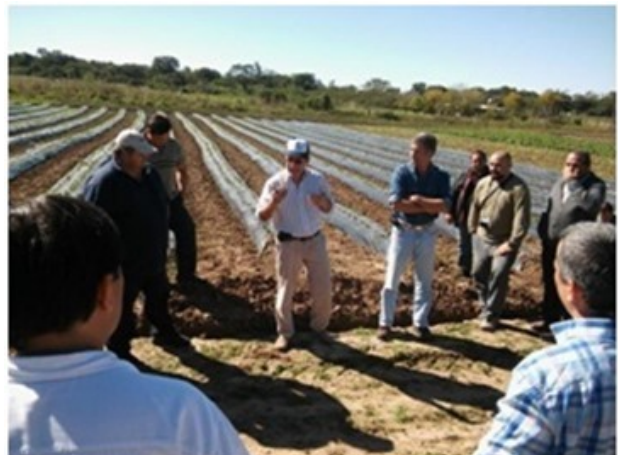

Figura 7. Grupo hortícola Viento Norte. Jornada a campo de producción de frutilla.

Los resultados obtenidos en el grupo apícola Humedales saludables (Figura 8). son los siguientes:

El 100\% implementa la técnica del Sendero Tecnológico propuesto por INTA PROAPI mediante la herramienta Unidad Demostrativa Apícola Orgánica (UDA).

El $67 \%$ incrementaron las colmenas dedicadas a la producción orgánica.

El $100 \%$ generaron agregado de valor a su producción, a través del fraccionamiento de miel orgánica, y disminuyeron sus costos gracias a la gestión en la compra en conjunto, de los insumos requeridos en el ciclo productivo.

El $89 \%$ se incorporó formalmente al sistema cooperativo, posibilitando mejorar el sistema organizativo institucional y las actividades de desarrollo territorial (Figura 9). 


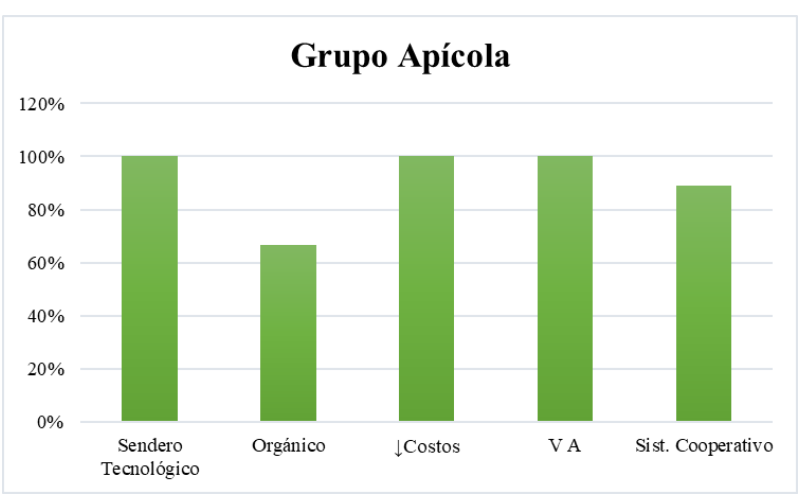

Fuente: Elaboración propia, a partir de datos PA Codutti, D.

Figura 8. Productores que incorporaron sendero tecnológico, cambio a producción orgánica, disminución de costos, valor agregado y participación en el sistema cooperativo ( $\%$ de un total de 10 productores).

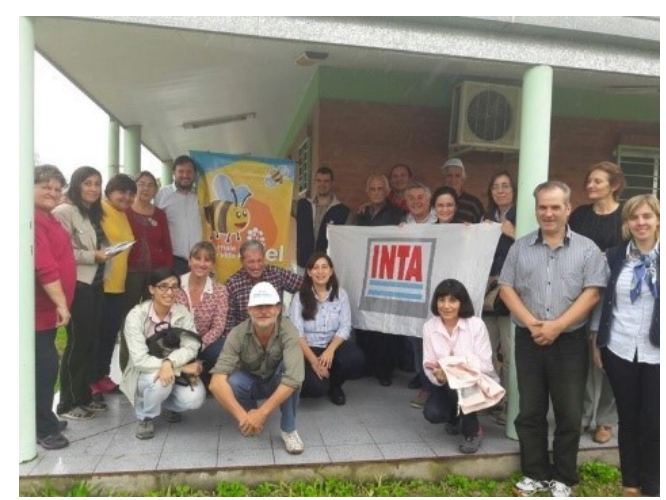

Figura 9: Grupo apícola Humedales Saludables. Reunión en la cooperativa apícola.

Los resultados obtenidos en el grupo Piscícola son los siguientes (Figura 10):

El 50\% incrementó y mejoró la comercialización (especies Tilapia y Pacú),

El 50\% incorporó tecnología en alimentación y cosecha.

El 50\% disminuyó sus costos productivos, a través de la compra en cantidad de insumos del proceso, (alimento balanceado).

Un 25\% generó valor agregado a su producción (fileteado, milanesas, molida).

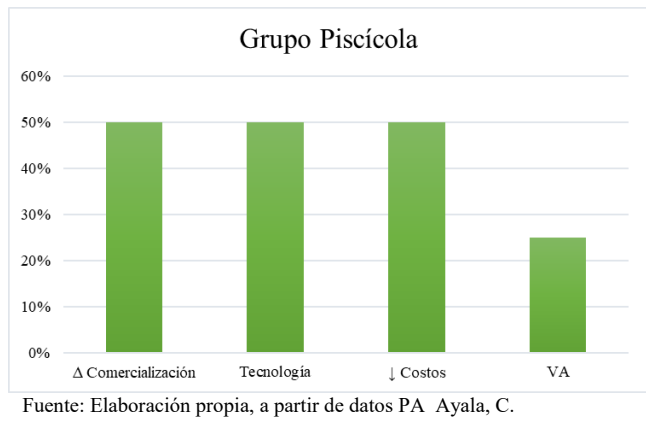

Figura 10. Productores que incorporaron tecnología, valor agregado, disminuyeron costos y mejoraron la comercialización ( $\%$ de un total de 10 productores).

Los resultados obtenidos en el grupo Porcinos del Este (Figura 11) son los siguientes:

El 100\% implementó un plan sanitario.

El 100\% se asoció a una Cooperativa de Productores Porcinos y lograron disminuir sus costos productivos, sincronizando sus compras de insumos (alimentos balanceados, sanidad).

El 88\% incrementó y mejoró la comercialización, realizándola de manera conjunta con el grupo en carnicerías de la zona.

El 75\% mejoró la nutrición de su producción y

Un 25\% logró valor agregado a través de elaboración de chacinados y chorizos.

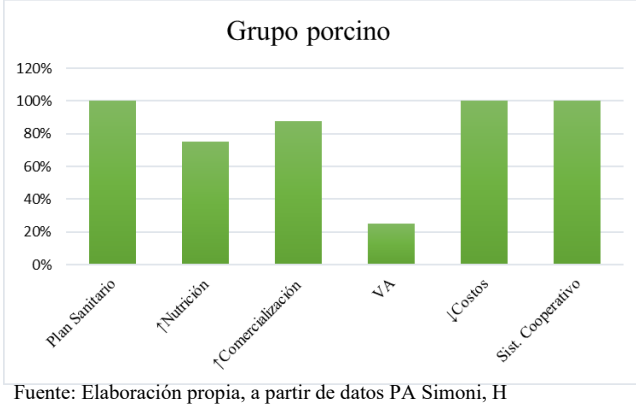

Figura 11. Productores que mejoraron el plan sanitario, nutrición, comercialización, disminuyeron costos, valor agregado y se incorporaron al sistema cooperativo (sobre 10 productores). 
Los resultados obtenidos en los productores de los grupos de turismo rural fueron (Figura 12):

El 100\% implementaron herramientas de marketing digital mejorando la promoción (Figura 13).

El 100\% generó vinculaciones con la Asociación de Prestadores de Servicios.

El 38\% incrementó el número de visitantes.

El 75\% mejoró el posicionamiento en su oferta de servicios.

El 25\% logro acceso al crédito.

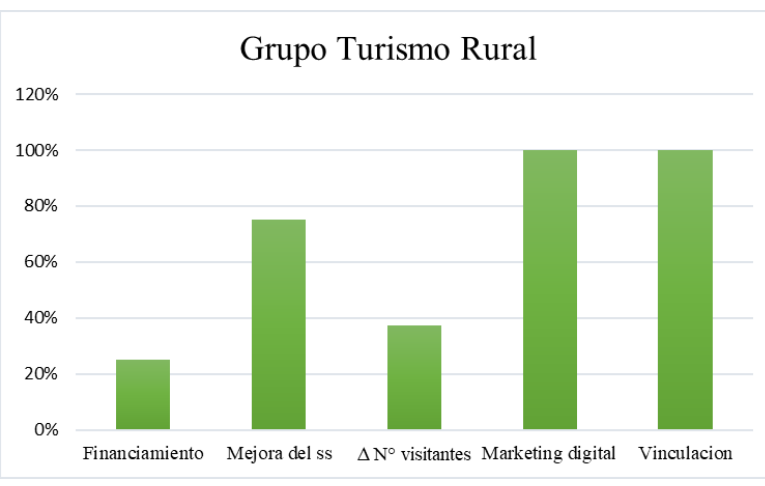

Fuente: Elaboración propia, a partir de datos PA Lencina.

Figura 12. Productores que mejoraron en vinculación, marketing, $\mathrm{n}^{\mathrm{o}}$ de visitantes, servicios y fuentes de financiamiento (sobre 40 productores).

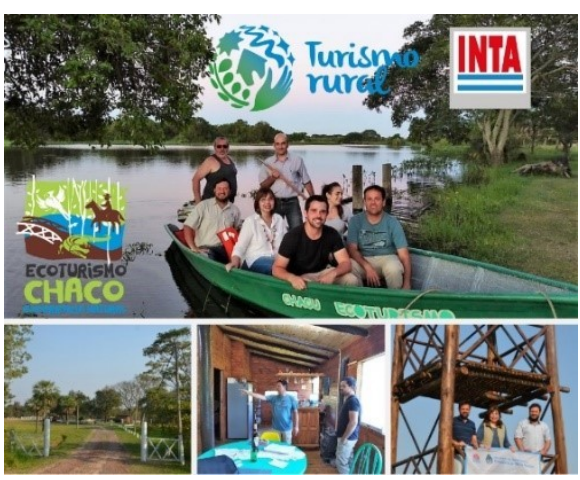

Figura 13. Grupo Ecoturismo Chaco.

\section{Participación en proyectos y articulación interinstitucional con aporte económico.}

Los proyectos formulados y aprobados con participación grupal fueron:

1. Proyecto de extensión del Programa Universidad en el Medio 0149-2016. Título "Fortalecimiento del Sector Apícola del Sudeste Chaqueño". Fecha de inicio: 01/01/2017-31/12/2017. Director: Cristina Salgado. Co-Director: Daniel Ruiz Díaz. Coordinador: Víctor Rusas. (Apoyo económico \$ 16.000).

2. Proyecto de extensión del Programa Universidad en el Medio Res.290-2018. Título "Sustentabilidad Apícola de los Humedales del Chaco: puesta en VALOR". Fecha de inicio: 25/04/2018-25/04/2019. Director: Cristina Salgado. Co-Director: Daniel Ruiz Díaz. Coordinador: Víctor Rusas. (Apoyo económico \$20.000).

3. Proyecto de extensión del Programa Universidad en el Medio Res.194-2019. Título " Mieles de Humedales: potencial florístico y calidad". Fecha de inicio: 01/05/2019-01/05/2020. Director: Cristina Salgado. CoDirector: Sandra Sobrado. Coordinador: Víctor Rusas. (Apoyo económico \$ 30.000).

4. Proyecto de extensión del Programa Universidad en el Medio Res.290/18. Título "Buenas prácticas de producción hortícola en Chaco y Corrientes" fecha de inicio 25/04/2018. Director Gladis Arce. Coordinador: German Pérez. (Apoyo económico \$20.000).

5. Proyecto de extensión del Programa Universidad en el Medio Promoción Res.194/19 Título "Buenas Practicas en sistemas productivos intensivos de Chaco y Corrientes". Fecha de inicio 10/04/19, Director: German Pérez. Co-Director Miriam Porta. (Apoyo económico \$30.000).

6. Convenio de colaboración tecnológica INTA con la Fundación Mujeres Apícolas 2018. Coordinador: Javier Vagabculov. (Apoyo económico \$45.000).

\section{CONSIDERACIONES FINALES}

A través del análisis de los datos disponibles y en virtud de los resultados obtenidos se pudo concluir que en este periodo de dos años (2018-2020), el rol del programa Cambio Rural como herramienta dinamizadora de procesos de desarrollo grupal y de extensión, fue sumamente importante. Los grupos de productores que más se apropian de la metodología del programa, más se potencian, generándose así el desarrollo en el territorio. En este sentido, se pudo ver que el grupo, se constituye como un importante soporte para la toma de decisiones, porque sostiene al productor, le ofrece un ámbito de reflexión sobre las ventajas y desventajas de las alternativas y lo acompaña en la ejecución y mantenimiento de las mismas. 
También, se pudo evidenciar que los grupos que lograron identificar el objetivo grupal con mayor facilidad son los que más rápido avanzaron en la resolución de problemáticas y en una gestión eficiente.

La mayoría de los productores integrantes de los grupos afianzaron sus fortalezas, accedieron a detectar y trabajar sobre los puntos débiles de sus actividades, y sus problemas, y pudieron aprovechar el esfuerzo y sinergia del conjunto; facilitados por la asistencia técnica del Promotor Asesor, el Agente de Proyecto y el apoyo del equipo técnico de extensión e investigación de la EEA Colonia Benítez del INTA.

En los grupos, quedaron instalados los logros comunes, a través del asociativismo lo que posibilitó o facilitó la incorporación de tecnologías adaptadas a cada actividad, comercializando productos y servicios en conjunto como así también se disminuyendo costos productivos, y consiguiendo agregar valor mediante nuevos productos, servicios y procesos.

Otra capacidad instalada en los grupos, es la vinculación institucional e interinstitucional lograda que permitió articular en diferentes niveles municipales, provinciales, interprovinciales, nacionales, con privados y organismos gubernamentales.

Desde el equipo técnico de Cambio Rural y de la EEA Colonia Benítez del INTA, se trabajó fuertemente en la aplicación de la herramienta metodológica para el armado de reuniones eficientes quedando la experiencia instalada a nivel grupo. Esto es muy importante, porque permitió capitalizar el tiempo de trabajo y la planificación de actividades, logrando resultados en función a los planes de trabajo y planes individuales, como herramienta fundamental para el desarrollo de los productores; finalidad primera del programa Cambio Rural.

\section{AGRADECIMIENTOS}

Se agradece muy especialmente a cada uno de los referentes de grupos por el compromiso asumido, a cada productor integrante y especialmente a cada Promotor Asesor por el desempeño en promover, facilitar y coordinar a cada grupo en el territorio.

A los profesionales pertenecientes al área de investigación y extensión del INTA Colonia Benitez por brindar el apoyo técnico en cada capacitación demandada, a la Coordinación Territorial y Nacional de Cambio Rural, al equipo de Agentes de Proyectos del NEA por brindar siempre acompañamiento e información.

Al equipo y cuerpo docente de la UNNE y proyecto UNNE en el Medio del área apícola y hortícola.

\section{BIBLIOGRAFÍA}

RIAN. (2014). Boletín RIAN Chaco-Formosa. Informe mensual agropecuario por Departamentos. Ediciones INTA. https://inta.gob.ar/sites/default/files/script-tmp-inta_informe_rian_chaco_formosa_diciembre2014.pdf.

Ministerio de Agroindustria de la Nación Secretaría de Agricultura, Ganadería y Pesca. Manual Operativo Cambio Rural. https://www.argentina.gob.ar/agricultura/extension-y-cambio-rural/manualoperativo, https://www.argentina.gob.ar/agricultura/extension-y-cambio-rural.

Morello, J. y Adámoli, J. (1974). Las grandes unidades de vegetación y ambiente del Chaco argentino. Segunda parte: Vegetación y Ambiente de la Provincia del Chaco. Serie Fitogeográfica N 13. INTA, Buenos Aires, $130 \mathrm{pp}$.

Ortegón, E., Pacheco, J.F. y Prieto, A. (2015). Manual - Metodología del marco lógico para la planificación, el seguimiento y la evaluación de proyectos y programas. CEPAL Serie Manuales. https:// repositorio.cepal.org/bitstream/handle/11362/5607/S057518_es.pdf

Rivière, P. E. (1985). El Proceso Grupal. Buenos Aires: Nueva Visión. 